\title{
Experiments Needed in Meson and Baryon Spectroscopy
}

\author{
D. V. Bugg \\ Physics Department, Queen Mary, University of London, London E1 4NS, UK \\ Correspondence should be addressed to D. V. Bugg, d.bugg@rl.ac.uk
}

Received 17 September 2007; Accepted 30 November 2007

Recommended by Peter J. Bussey

Three (or four) straightforward experiments would contribute greatly to completing the spectroscopy of baryons and light mesons. In the baryon sector, data are needed on inelastic reactions from a polarised target with $\pi^{ \pm}$and $K^{ \pm}$beams up to $\sim 3 \mathrm{GeV} / \mathrm{c}$. Similar data are needed in the light meson sector for $\bar{p} p$ interactions in the momentum range $0.3-2 \mathrm{GeV} / \mathrm{c}$. In both cases, valuable information is to be obtained from longitudinal (L) and sideways (S) target polarisations as well as the conventional normal (N) polarisation. Thirdly, ${ }^{3} S_{1}$ and ${ }^{3} D_{1}$ mesons in the mass range $1-2.4 \mathrm{GeV}$ could probably be separated either by diffractive dissociation of transversely polarised photons or by $e^{+} e^{-}$radiative return experiments using transversely and longitudinally polarised electrons.

Copyright (C) 2007 D. V. Bugg. This is an open access article distributed under the Creative Commons Attribution License, which permits unrestricted use, distribution, and reproduction in any medium, provided the original work is properly cited.

\section{Introduction}

This is a discussion document aiming at stimulating discussion of a fresh round of high-quality experiments on baryon and meson spectroscopy. QCD is widely believed to lead to confinement of mesons and baryons. Lattice Gauge calculations are now possible with unquenched quarks. The opportunity exists to confront these calculations with as complete a spectrum as possible of experimentally observed states. The experiments could be done quite cheaply and quickly.

This is not just "stamp-collecting," as some cynics claim. Without such data, it is not possible to say exactly how QCD really works in the nonperturbative regime. Understanding confinement is a key issue in particle physics, but it is being neglected. Confinement is clearly a phase transition, but it is quite possible that it exhibits similar subtlety to chemistry and solid state physics. Chiral symmetry breaking is also clearly a phase transition. Its relation to confinement needs to be understood. Perhaps they are the same phase transition; perhaps they are related in a more subtle way. 
Hybrids and glueballs are expected, but without a complete picture of quark-model states, progress in identifying them is frustrated. What role, if any, do glueballs play in the confinement process? The baryon and light meson sectors are the ones where it is presently technically feasible to achieve a complete spectroscopy or something close.

Many $N^{*}$ and $\Delta$ resonances are known up to $\sim 2200 \mathrm{MeV}$ [1]. They are readily interpreted as 3-quark states. However, the spectrum is incomplete. Low spin states are missing, or poorly identified. No member of the SU6 $\{20\}$ multiplet is firmly established, although there are candidates; perhaps they do not exist.

Present baryon results come largely from experiments in the 1960-1970 era using liquid hydrogen and polarised targets. There are also good data on $\pi^{-} p \rightarrow \eta n$ [2] and a little on $\omega n$ [3]. The Crystal Ball collaboration has produced data on $\pi^{-} p \rightarrow \pi^{0} \pi^{0} n$ [4] and $K^{-} p \rightarrow \pi^{0} \pi^{0} \Lambda$ $[5]$.

Data on further inelastic channels come from bubble chamber experiments but with low statistics; these have been analysed by Manley and Saleski [6]. There are also low statistics data on $\Lambda K$ and $\Sigma K$ final states, including polarisation information from hyperon decays [7]. Experiments on photoproduction are beginning to make decisive contributions [8-11] but the photon spin complicates the analysis. The CLAS collaboration at JLAB proposes to take data with polarised photons and a polarised target. This will augment existing data [12] and strengthen the partial wave analysis considerably. Complementary information from $\pi N$, with its simpler spin structure, would strengthen this partial wave analysis, and would also isolate couplings specific to photons.

Because of the spin $1 / 2$ of the nucleon, it is essential to have polarisation data. These data also fulfill a second important role. Differential cross sections depend on intensities of partial waves and the real parts of interferences between them. The quantity $P d \sigma / d \Omega$ depends on $\operatorname{Im} f^{*} g$, where $f$ is the spin-averaged amplitude and $g$ the spin-flip amplitude. It is therefore phase sensitive and plays a key role in establishing the phase variation of amplitudes.

In the near future, several major $4 \pi$ detectors will complete their current programmes: Belle, Babar, Cleo C, and Kloe. They are superb detectors which have much to offer for the experiments proposed here. The one new feature which is required is a frozen spin polarised target. The technology of such targets is well developed and costs a small fraction of the detectors themselves. Rather than scrapping these detectors or cannibalising them, why not put some of them to use on a new programme of spectroscopy? The statistics required are modest, so the required data could be collected quite quickly.

The partial wave analysis would be the bigger problem. Why not harness the efforts of the army of phenomenologists who speculate on how QCD works to doing the partial wave analysis and finding out how it really does work? This would be a welcome return to an earlier generation where experimentalists and phenomenologists worked hand in hand.

In the light meson sector, the spectrum is incomplete around $1600-1700 \mathrm{MeV}$. In the mass range 1910-2400 MeV, data from LEAR provide a complete spectrum of $I=0, C=+1$ states, summarised in the final coupled-channel analysis of [13]. This spectrum is currently not listed in the regular part of the Particle Data Book, because this is the only experiment to observe most of the states; results are to be found on [1, pages 644-648]. It is important to realise that many of the states have been identified in as many as 7 independent sets of data. This makes identification of resonances extremely secure: it can be shown by analysing sub-sets of these data that the confidence level increases roughly as 2 to the power of the number of data sets. 


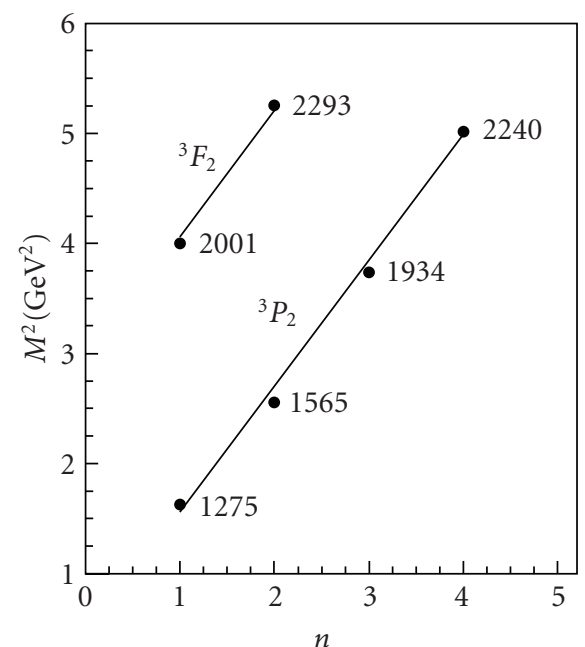

(a)

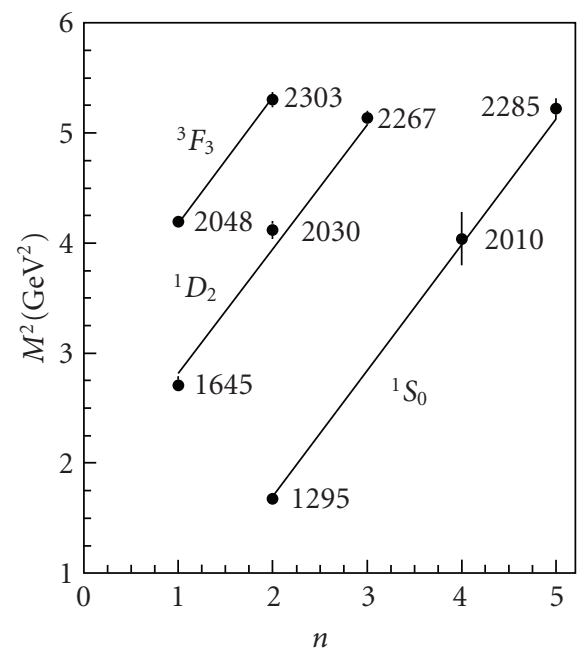

(c)

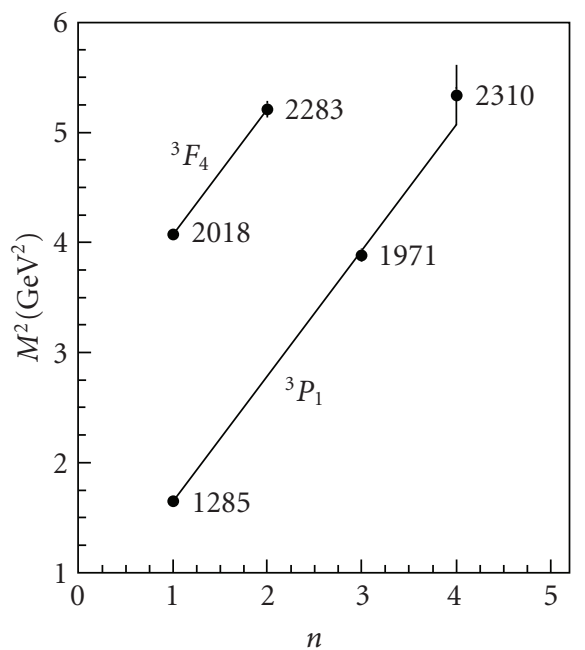

(b)

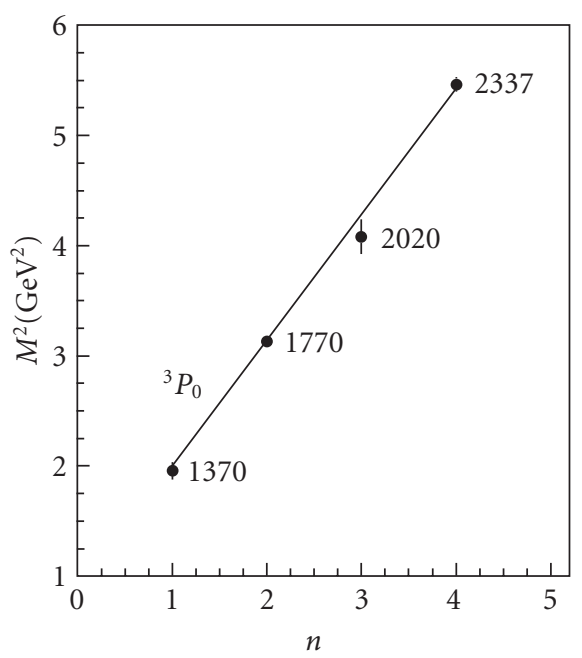

(d)

Figure 1: Trajectories of $I=0, C=+1$ light mesons; $n$ is the principal (radial) quantum number and $n=1$ for ground-states; $M$ is the mass and points (with errors) show known states while numbers indicate masses in $\mathrm{MeV}$.

For $J^{P}=0^{+}, 2^{+}$and $4^{+}$, the combined fit has a confidence level better than the best individual set by a factor 60 . This multiplicity of final states needs to be studied in baryon spectroscopy.

Figures 1, 2, and 3 show the known light mesons above $1 \mathrm{GeV}$ [14]. They fall into a simple pattern of parallel Regge trajectories. Klempt has drawn attention to the fact that $N^{*}$ and $\Delta$ states fall on similar trajectories of almost the same slope, but with larger errors [15].

An intriguing feature of both meson and baryon spectra is the appearance of parity doublets: states with the same isospin $I$ and spin $J$, but opposite parity $P$. It is a feature of QCD that it is $\mathrm{SU}(3)_{L} \otimes \mathrm{SU}(3)_{R}$ symmetric if quark masses are negligible. However, it is well known that this symmetry is spontaneously broken for the lowest states. Firstly, the nucleon has no nearby $J^{P}=(1 / 2)^{-}$partner. Secondly, in the meson sector, well identified Adler zeros in $\pi \pi$ and $K \pi$ elastic scattering arise from chiral symmetry breaking. 


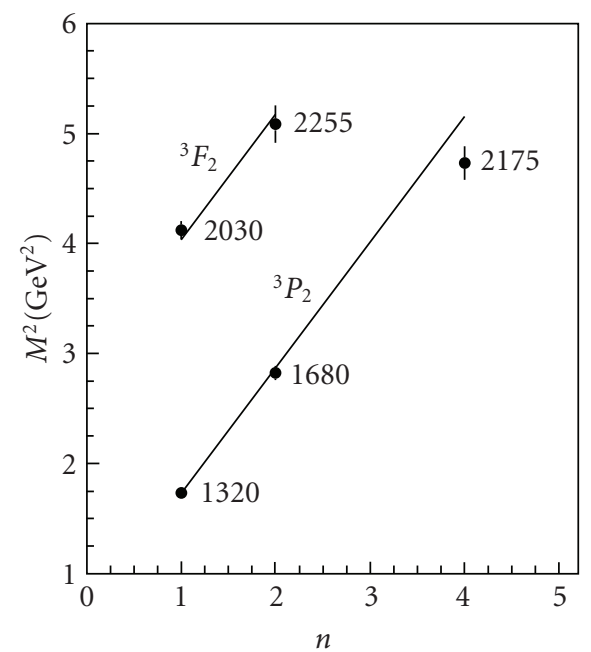

(a)

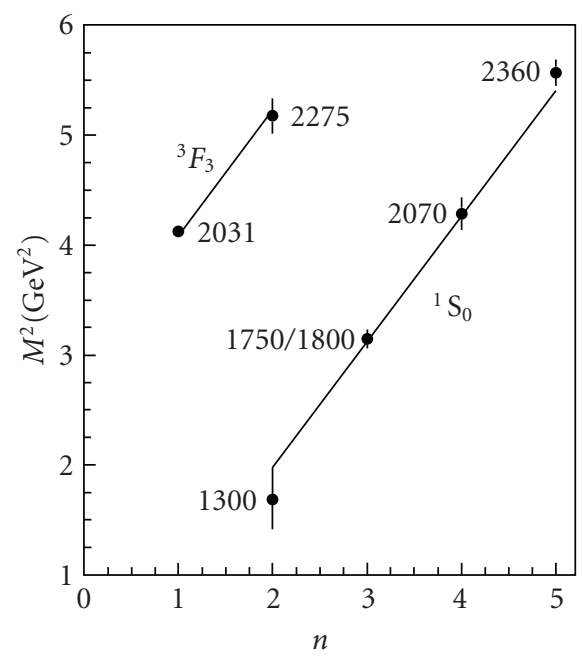

(c)

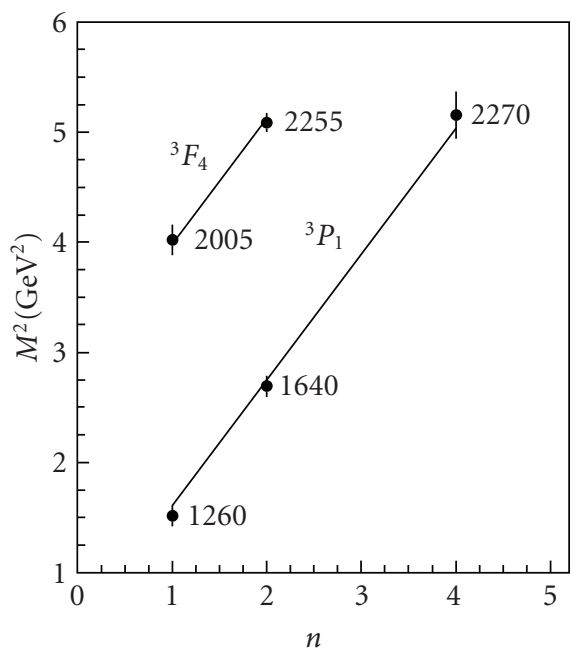

(b)

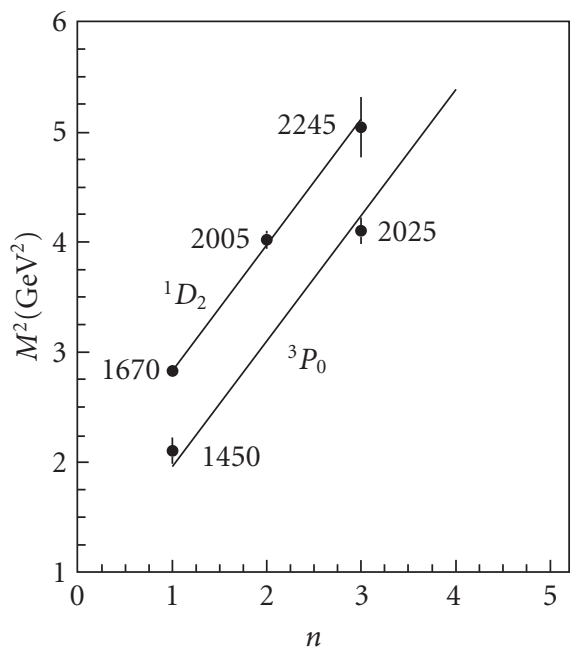

(d)

Figure 2: Trajectories of $I=1, C=+1$ states [14].

Glozman [19] proposes in a series of papers that chiral symmetry is approximately restored high in the spectrum. This would require $J^{P C}=4^{+-}$mesons at masses close to the well known $4^{++}$states $f_{4}(2040)$ and $a_{4}(2040)$. Likewise $3^{+}$partners are needed for the well known $3^{-}$ ground-states $\rho_{3}(1690)$ and $\omega_{3}(1670)$. However, these states are currently missing [20], raising questions about this scheme. Jaffe also comments on the question of chiral symmetry restoration [21, 22]. Shifman and Vainstein present disagreements with Glozman's scheme [23].

A feature of states labelled ${ }^{3} P_{2}$ on Figure 1 is that they lie systematically lower in mass than those labelled ${ }^{3} F_{2}$ by $\sim 80 \mathrm{MeV}$. The labelling arises from the fact that ${ }^{3} F_{2}$ states (a) decay mostly with $L=3$ and (b) are nearly degenerate with ${ }^{3} F_{3}$ and ${ }^{3} F_{4}$ mesons. Likewise, $D$ states lie systematically above $P$ states by $\sim 40 \mathrm{MeV}$. However, Glozman objects that orbital angular momentum should not be a good quantum number for a rapidly rotating string with highly relativistic quarks attached to each end. Instead $J$ should be the good quantum number [24]. 


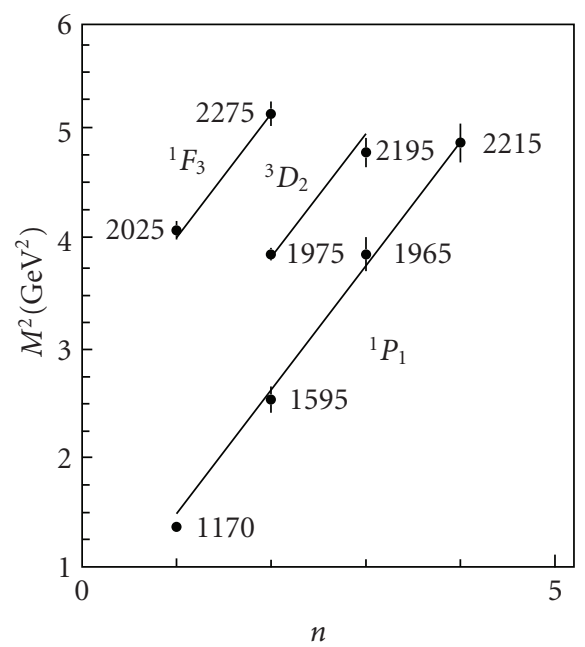

(a)

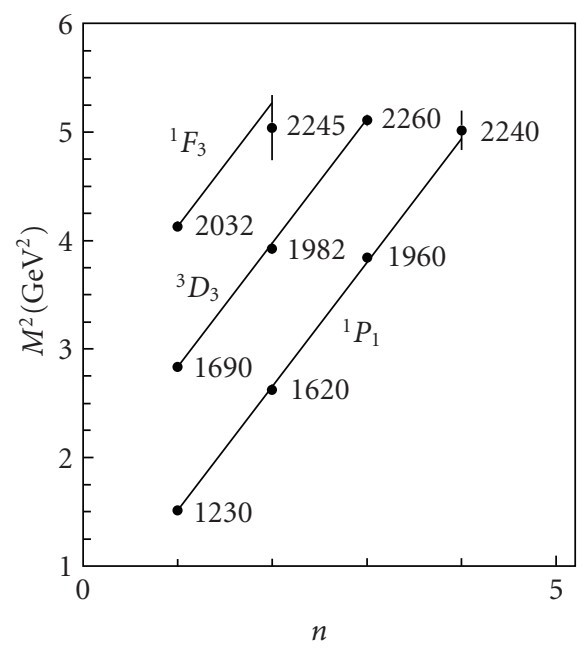

(c)

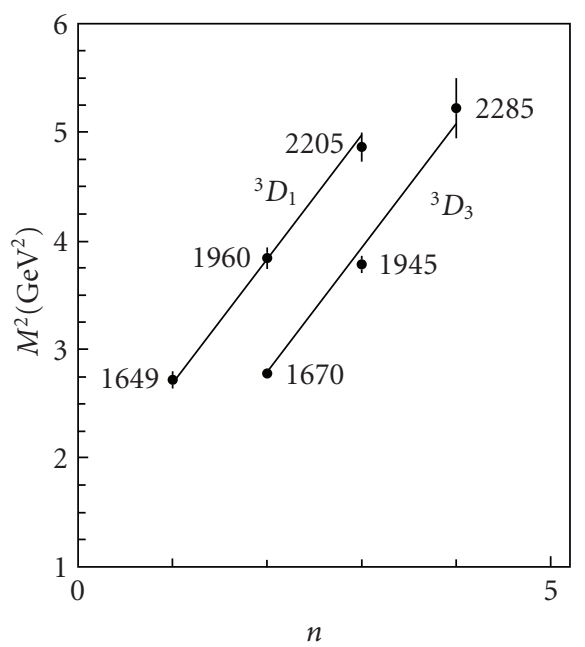

(b)

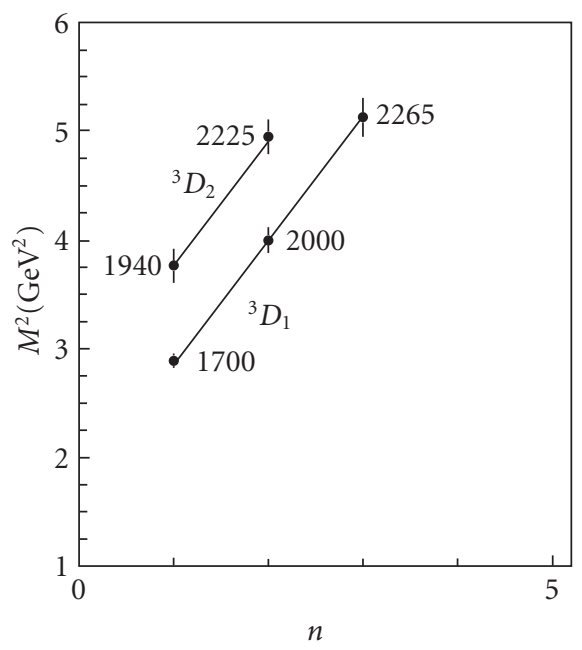

(d)

Figure 3: Trajectories of $C=-1$ states with (a) and (b) $I=0$, (c) and (d) $I=1$. In (b), the ${ }^{3} D_{3}$ trajectory is moved one place right for clarity; in (d), ${ }^{3} D_{2}$ is moved one place left [16-18].

Afonin [25] provides an excellent general review of the history and details of the spectroscopy. He arrives at a different scheme where states fall more naturally into hydrogen-like representations of the dynamical $O(4)$ group. This difference from Glozman immediately illustrates the fact that better and more complete data are required to settle even the general features of how QCD actually works. Afonin also traces a very interesting connection of MacDowell symmetry from the baryon to the meson sector [26]. Experiment might provide a useful guideline to methods of approximation in lattice Gauge calculations.

The light $1^{--}$mesons are particularly poorly identified. The essential reason for this is that each state has a width $\geq 250 \mathrm{MeV}$, but ${ }^{3} S_{1}$ and ${ }^{3} D_{1}$ states overlap at mass intervals less than this. If Crystal Barrel data are a guide, ${ }^{3} S_{1}$ states should decay dominantly with $L=0$ in the final state (e.g., to $\left[b_{1}(1235) \pi\right]_{L=0}$ ) while ${ }^{3} D_{1}$ states should decay dominantly with $L=2$. If so, an experiment on diffraction dissociation of transversely polarised photons should make 
a clear distinction between ${ }^{3} S_{1}$ and ${ }^{3} D_{1}$ states. For $D$-wave decays, the partial wave decomposition is

$$
|1,1\rangle=\sqrt{\frac{6}{10}}|2,2\rangle_{L}|1,-1\rangle-\sqrt{\frac{3}{10}}|2,1\rangle_{L}|1,0\rangle+\sqrt{\frac{3}{10}}|2,0\rangle_{L}|1,1\rangle
$$

A linearly polarised photon is a superposition of initial states $|1,1\rangle$ and $|1,-1\rangle$ with the result that interference terms appear, generating distinctive dependence on the azimuthal angle $\phi$ from the plane of polarisation, through terms depending on $\phi$ and $2 \phi$.

A linearly polarised photon beam of $9 \mathrm{GeV}$ is planned for the GlueX experiment at JLab [27]. It should be possible to adapt this beam for photons over the range 1 to $2.5 \mathrm{GeV} / \mathrm{c}$. There is an excellent prospect that this would identify individual ${ }^{3} S_{1}$ and ${ }^{3} D_{1}$ states cleanly.

The alternative (or a complementary experiment) is to use so-called radiative return in $e^{+} e^{-}$scattering using polarised electrons. One hard photon is radiated from the initial state and the surviving $e^{+} e^{-}$pair interacts via a photon to generate $J^{P}=1^{--}$final states. It is desirable to use both linearly and longitudinally polarised electrons, to distinguish clearly between $L=2$ and $L=0$ decays of resonances.

So far, the discussion has centred on light mesons and baryons. There has, of course, been spectacular progress on the spectroscopy of charmed mesons ( $D$ and $D_{s}$-mesons) and baryons and the family of states with hidden charm, $J / \Psi$, and so forth. B-factory data measure the spins of hyperon states from charmed baryon decays; this has contributed strongly to identifying several charmed baryons and also to identifying several $\Xi$ states. Data from decays of $B$-mesons is contributing to the study of charmed mesons, and also the study of light $q \bar{q}$ states and hybrid candidates such as $Y_{2}(4260)$.

Lattice calculations are easier for heavier quarks. If the spectroscopy of these states could be extended to broad states and radial excitations, the spectrum would be easier to compare with Lattice calculations. But presently such data seem a remote prospect. My essential message is that spectroscopy of light mesons and baryons is a practical proposition over a relatively short time-scale. Perhaps, with this extra information, the spectroscopy of charmed states would become clearer, at least one could ask different questions.

\section{Practical considerations}

In 1989, David Axen and I made a detailed study of a possible experiment on $\Lambda^{*}, \Sigma^{*}$, and $\Xi^{*}$ spectroscopy [28] using a polarised target. This paper is not available online; copies are available from the authors. An outline of the contents of the paper will be given here.

It was written in the context of the $K$-factory being discussed at the time. It considered 15 readily accessible $K^{-} p$ reactions. The study assumed a detector close to $4 \pi$ acceptance and good $\gamma$ detection. It showed that it is realistic to increase bubble chamber statistics by 2 to 3 orders of magnitude.

The design of the polarised target is simplified greatly if the detector is used without a large-scale magnetic field. The orientation of the target spin may then be manipulated simply by means of holding coils of $\sim 0.25 \mathrm{~T}$. A target length of $4 \mathrm{~cm}$ is realistic and a diameter $\leq 1 \mathrm{~cm}$; the holding coils then have dimensions $\sim 2 \mathrm{~cm}$ larger than the target in each direction. The holding field bends charged particles by modest amounts, but trajectories can be reconstructed iteratively. 
Table 1: Easy channels for baryon spectroscopy; the upper part of the table assumes particle momenta are unmeasured; the lower part assumes neutron time-of-flight is measured.

\begin{tabular}{|c|c|c|}
\hline$\pi^{+} p \rightarrow \pi^{+} \pi^{0} p$ & $2 \mathrm{C}$ & (1) \\
\hline$\rightarrow \pi^{+} \eta p$ & $2 \mathrm{C}$ & (2) \\
\hline$\rightarrow \pi^{+} \omega p$ & $2 \mathrm{C}$ & (3) \\
\hline$\rightarrow \pi^{+} \pi^{0} \pi^{0} p$ & $2 \mathrm{C}$ & (4) \\
\hline$\rightarrow \Sigma^{+} K^{+}$ & $3 C$ & (5) \\
\hline$\pi^{-} p \rightarrow \pi^{-} \pi^{0} p$ & $2 \mathrm{C}$ & (6) \\
\hline$\rightarrow \eta n$ & $3 C$ & $(7)$ \\
\hline$\rightarrow \omega n$ & $3 C$ & (8) \\
\hline$\rightarrow \eta^{\prime} n$ & $3 C$ & (9) \\
\hline$\rightarrow \pi^{-} \eta p$ & $2 \mathrm{C}$ & (10) \\
\hline$\rightarrow \pi^{-} \omega p$ & $2 \mathrm{C}$ & (11) \\
\hline$\rightarrow \pi^{0} \pi^{0} \pi^{0} n$ & $3 C$ & (12) \\
\hline$\rightarrow \Lambda K^{0}$ & $2 \mathrm{C}$ & (13) \\
\hline$\rightarrow \Sigma^{0} K^{0}$ & $2 \mathrm{C}$ & (14) \\
\hline$\rightarrow \Sigma^{-} K^{+}$ & $2 \mathrm{C}$ & (15) \\
\hline$\pi^{+} p \rightarrow \pi^{+} \pi^{+} n$ & $2 \mathrm{C}$ & (16) \\
\hline$\pi^{-} p \rightarrow \pi^{+} \pi^{+} \pi^{0} n$ & $2 \mathrm{C}$ & (17) \\
\hline$\rightarrow \pi^{-} \pi^{+} n$ & $2 \mathrm{C}$ & (18) \\
\hline$\rightarrow \pi^{-} \pi^{+} \pi^{0} n$ & $2 \mathrm{C}$ & (19) \\
\hline
\end{tabular}

Such a target operated at Triumf in the late 1980's and an operating temperature of $0.06^{\circ} \mathrm{K}$ was achieved. It may be possible to improve on that today. Under those conditions, the relaxation time of the target polarisation was typically 4 days. The initial polarisation which was achieved was $85 \%$. The beam needs to enter along the cryostat of the polarised target. This cryostat needs to be withdrawn from the detector from time to time into a very uniform field of $2.5 \mathrm{~T}$, where the target can be polarised by the usual techniques.

\section{Baryon spectroscopy}

Table 1 shows a selection of $\pi p$ reactions which should be straightforward from a polarised target without a magnetic field. Column 3 shows the number of kinematic constraints which are available if momenta are not measured. It is assumed that photons are detected with the energy and position resolution of typical $\mathrm{CsI}$ or $\mathrm{BaF}_{2}$ detectors today. The energies and directions of $\pi^{0}, \eta$ and $\omega$ (from $\pi^{0} \gamma$ ) are reconstructed fully. The $\eta^{\prime}$ may be detected via $\gamma \gamma$ or $\eta \pi^{0} \pi^{0}$ decays with similar efficiency and resolution.

It is assumed that momenta of charged particles are not measured, but $\pi, K$ and $p$ may be separated by $d E / d x$ and/or time-of-flight. In reactions (1)-(3), there are two unmeasured momenta, hence a $2 \mathrm{C}$ fit after using energy-momentum balance for the production reaction. Reaction (4) uses $\Sigma^{+} \rightarrow \pi^{0} p$. There are 2 unmeasured momenta, but 4 constraints from energymomentum conservation in the production process plus a further constraint from the relation between momentum and energy from the $\Sigma^{+}$decay; $K^{+}$identification makes this final state distinct. 
Similar arguments give the number of constraints shown in the table. For reactions (7)(15) it is assumed that neutrons convert in the $4 \pi$ detector, but without good time-of-flight information. This situation could be improved using a dedicated time-of-flight spectrometer at a distance of $\sim 2.5 \mathrm{~m}$, covering $0-70^{\circ}$ lab angles. The event rate would drop accordingly, but beam intensity is not a limitation and the trigger is simple. For reactions (16)-(19) it is assumed that neutron time-of-flight is measured. There is a physics reason why it may be desirable to obtain limited information on these channels, see Section 5.1.

With a $K^{-}$beam of the same modest intensity, it is possible to run a corresponding experiment on strange baryons. Here, a special trigger for all neutral final states could be used to investigate rare channels like $\Xi^{0} K^{0}$ and $\Xi^{0} K^{0} \pi^{0}$. It would also be of interest to run a $K^{+}$beam in case there really are pentaquarks.

A Monte Carlo study of the background from carbon or nitrogen atoms in the target estimates background levels of $10 \%$. However, it is straightforward to measure this background using a dummy target without hydrogen. The separation of hydrogen events may be estimated roughly in the following simple way. Nucleons in carbon have a Fermi momentum of $\sim 200 \mathrm{MeV} / \mathrm{c}$, that is, $115 \mathrm{MeV} / \mathrm{c}$ along each of $x, y$ and $z$-axes. Momenta of final state particles need to be constructed with an accuracy substantially better than this; $20 \mathrm{MeV} / \mathrm{c}$ is realistic. For example the error for $\pi^{0}$ reconstructed from photon pairs, after using the constraint on the $\pi^{0}$ mass, is on average better than this. Many of the reactions considered below have 2 constraints from energy-momentum balance, so an elementary guess for background levels is $3 \%$; in practice, difficult configurations make the backgrounds somewhat worse.

The conclusions of the 1989 Monte Carlo study were that

(1) reactions with integrated cross sections $\geq 1 \mathrm{mb}$ may readily be separated by $2 \mathrm{C}$ fits with a level of cross-talk between them and with other reactions generally in the 0.5 $2.0 \%$ range;

(2) the same is true for $3 \mathrm{C}$ fits to reactions with integrated cross sections $\geq 10 \mu \mathrm{b}$;

(3) these levels of cross-talk are less than or equal to those following from second scattering of exit particles in the material of the polarised target and in the detectors, that is, intrinsic experimental limits;

(4) vertices of $\Sigma^{ \pm}, \Xi$, and $K^{0}$ decays are identified accurately by tracking the charged particles.

The Monte Carlo study is backed up by experience in two experiments at Triumf [29] and Lampf [30] on the inelastic reaction $p p \rightarrow p n \pi^{+}$. There, backgrounds averaging $8 \%$ were observed, and could be measured to $<1 \%$.

Experience with Crystal Barrel is that statistics of $50 \mathrm{~K}$ events per channel are adequate for partial wave analysis. Suppose one aims for this with channels having an integrated cross section of $1 \mathrm{mb}$. This implies collecting a total of $\sim 6 \times 10^{6}$ events per momentum, allowing for the fact that roughly half come from carbon (or nitrogen). The Crystal Barrel experiment had a data acquisition rate of 60 events per second. For more complex detectors, data acquisition rate may be a problem, but the technology of LEP experiments is a huge step forward. Suppose an event rate of 100 events/s is possible. Then 6 million $\pi p$ events can be recorded in $<24$ hours of running time. With a $4 \mathrm{~cm}$ target length and a $30 \mathrm{mb}$ average total cross section, the required beam intensity is only 500 per second. Using steps of $30 \mathrm{MeV} / \mathrm{c}$ from 500 to $3000 \mathrm{MeV} / \mathrm{c}$, the total running time for 80 momenta is a few months per target spin orientation. There is useful 
Table 2: Channels to be studied in $\bar{p} p$ interactions.

\begin{tabular}{lccc}
\hline$I=0, C=+1$ & $I=1, C=+1$ & $I=0, C=-1$ & $I=1, C=-1$ \\
\hline$\pi^{0} \pi^{0}, \eta \eta, \eta \eta^{\prime}$ & $\eta \pi^{0}, \eta^{\prime} \pi^{0}$ & $\omega \eta$ & $\omega \pi^{0}$ \\
$\eta \pi^{0} \pi^{0}, \eta^{\prime} \pi^{0} \pi^{0}$ & $3 \pi^{0}, \eta \eta \pi^{0}$ & $\omega \pi^{0} \pi^{0}$ & $\omega \eta \pi^{0}$ \\
\hline
\end{tabular}

information from all three orientations of target spin, so one is talking about a total of 1 year of data collection.

A technical point is that it is necessary to monitor dead-times in the detector and dataacquisition system carefully. This is well known to be a systematic problem with most large detectors.

Experience at the PS172 experiment at LEAR was that differential cross sections can be deduced accurately from data on a polarised target. So it is not strictly necessary to run separately from a liquid hydrogen target, though some systematic cross-checks are desirable. Some running from liquid hydrogen is needed for the calibration of neutron detection efficiency.

\subsection{Three target polarisations}

It seems not to be realised widely that there is useful information from target polarisations in sideways (S) and longitudinal (L) directions. For elastic scattering, where all the particles lie in one plane, asymmetries from these spin orientations are zero. However, when the target spin is out of the plane of the final state, asymmetries are nonzero. These were measured in the Lampf experiment on $p p \rightarrow p n \pi^{+}$[30] and gave valuable information. Section 4 of that publication explains how to obtain formulae for traces involving the Pauli matrices $\sigma$ representing target polarisation. For hyperon decays in the final state, there is further information from traces involving spin operators for both target and hyperon. The most useful data are for transverse target polarisation, which gives information from real parts of interferences between partial waves, but with signs different from differential cross sections. Longitudinal target polarisations measure moduli squared of partial waves, but with signs which depend on the sign of $L$.

\section{Light meson spectroscopy}

The Crystal Barrel experiment studied 16 final states containing only photons. Background levels were as low as 1\% for the strongest channels, rising to $20 \%$ in the worst cases. However, there was so much physics information that the cross-talk between channels could be estimated reliably. Straightforward techniques were evolved to evaluate cross-talk arising from 45 channels which were separated at the stage of data-processing, although not all were used for physics, see [14, Section 2.2].

For meson spectroscopy above $1900 \mathrm{MeV}$, the essential idea is to study s-channel resonances:

$$
\bar{p} p \longrightarrow \text { Resonance } \longrightarrow A+B .
$$

Channels which needs studying are shown in Table 2. All of these final states are experimentally easy to identify using decays of $\omega$ to $\pi^{0} \gamma, \eta \rightarrow 2 \gamma$ and $3 \pi^{0}, \eta^{\prime} \rightarrow 2 \gamma$ and $\eta \pi^{0} \pi^{0}$. 
It is of the greatest importance to obtain data at the lowest available beam momenta. The PS172 polarised target ran as low as $360 \mathrm{MeV} / \mathrm{c} \equiv 1910 \mathrm{MeV}$ mass. The intention was to run the Crystal Barrel experiment likewise at $750 \mathrm{MeV} / \mathrm{c}$ and with $360 \mathrm{MeV} / \mathrm{c}$ at the target centre after energy loss. However, LEAR closed before the allocated beam time was delivered to the experiment, with the result that the lowest momentum was $600 \mathrm{MeV} / \mathrm{c} \equiv 1962 \mathrm{MeV}$. Since there is a cluster of resonances in the mass range $1920-2050 \mathrm{MeV}$, this was a serious loss to the experiment.

For $I=0, C=+1$, the analysis is already very tightly constrained for $J^{P}=0^{+}, 2^{+}$and $4^{+}$. These partial waves appear in seven sets of data: differential cross sections and polarisations in $\bar{p} p \rightarrow \pi^{+} \pi^{-}[31,32]$, and Crystal Barrel data on $\pi^{0} \pi^{0}, \eta \eta, \eta \eta^{\prime}, f_{2}(1270) \eta$ and $a_{2}(1320) \pi$. Phase information on other $J^{P}$ comes from their interferences with these states in $\eta \pi^{0} \pi^{0}$ and $\eta^{\prime} \pi^{0} \pi^{0}$ channels (and $\eta \eta \eta$ ). Polarisation for these channels would make the partial-wave analysis completely unambiguous. Incidentally, the data and analysis programmes and fitting parameters are publicly available on CDs from Sarantsev or myself. Partial waves are expressed in tensor algebra. An important cross-check on programmes is that all partial waves have been shown to be orthogonal when integrated over phase space.

For $I=1, C=-1$, the polarisation information from $\bar{p} p \rightarrow \pi^{+} \pi^{-}$already makes the current $q \bar{q}$ spectrum almost complete and rather well defined, except for $J^{P C}=1^{--}$, where overlap between ${ }^{3} S_{1}$ and ${ }^{3} D_{1}$ states causes confusion. For the other two sets of quantum numbers, the situation is presently not nearly so well defined, because of the lack of any polarisation data. Results are consistent with spectra close to those of the other two families, but several states are missing and there is significant flexibility in the solutions. Indeed two solutions exist for $2^{+}$ and $4^{+}$partial waves for $I=1, C=+1$, though one solution is significantly better. Simulations with current data show that polarisation information on $\eta \pi, 3 \pi^{0}, \omega \pi^{0}, \omega \eta, \omega \pi^{0} \pi^{0}$ and $\omega \eta \pi^{0}$ channels would make the analyses of $q \bar{q}$ states completely unambiguous.

For the $3 \pi^{0}$ channel, there is the possibility that the $\pi \pi I=2$ channel could contribute. (This is forbidden from initial $\bar{p} p$ states with $I=1$ and 0 in all other cases.) There is currently no evidence for $I=2 \pi \pi$, but that needs confirmation.

This proposed experiment is not part of the PANDA programme at FAIR [33] which will run with $\bar{p}$ from $1.5 \mathrm{GeV} / \mathrm{c}$ to $15 \mathrm{GeV} / \mathrm{c}$ on liquid hydrogen. It would however be feasible at the lower momentum ring FLAIR if there is physical space to accomodate a large detector. The existing Crystal Barrel detector is adequate for the task. A beam intensity of a few $\times 10^{4}$ is needed with a trigger on all neutral final states. Other detectors have charged particle detection, which does not survive in the present incarnation of the Crystal Barrel. In reactions with charged pions in the final state, $G$ is the relevant quantum number rather than $C$. Accordingly, there are interferences between $I=1$ and $I=0$ states, providing very delicate cross-checks on parameters of resonances in these two systems.

\section{Partial wave analysis}

There is a difference of opinion between experimental groups as to whether to do so-called Energy Independent Analysis or Energy Dependent Analysis. In the former case, amplitudes are fitted freely in magnitude and phase in every mass bin. In the latter case, all partial waves are parametrised with analytic forms. In practice this means Breit-Wigner resonances plus backgrounds linear in $s$, if needed. 
The problem with the latter is that one is actively putting resonances into the analysis, rather than "deducing" them from the behaviour of amplitudes on the Argand diagram. The converse problem with Energy Independent Analysis is that it is theoretically impossible without polarisation information. In one bin, there is only one piece of information: a differential cross section. It is impossible in principle to derive both magnitudes and phases of all amplitudes. Energy Independent analysis therefore proceeds by parametrising some well known resonances with Breit-Wigner amplitudes, and using them as interferometers to determine other partial waves. This works well for the $\rho$ and $\pi_{2}(1670)$, whose parameters are well known. However, for other resonances there are problems. One example will suffice. It is common practice to use the $f_{2}(1270)$ as an interferometer. However, one usually finds with good data that the $f_{2}(1565)$ is produced with it. Its line-shape is not well known, because of lack of data on the dominant $4 \pi$ channel; it overlaps significantly with $f_{2}(1270)$. Worse, it is known to couple strongly to $\omega \omega$. The subthreshold continuation of this channel can introduce serious distortions in what is fitted as $f_{2}(1270)$.

The advantage of Energy Dependent analysis is that data can be fitted simultaneously from a set of reactions. In energy independent analysis, this constraint is lost unless constraints from other data are imposed by fixing resonance mass, width, and/or branching ratios. When a weak signal is analysed, this type of constraint is important. For the case of multibody final states, for example, $4 \pi$, a combination with analysis of 2-body channels is the only reliable approach.

Experience in both $\pi N$ and $\bar{p} p$ analyses is that the constraint of analyticity plays a vital role, even when polarisation data are available. If this constraint is not applied, partial waves rapidly deviate from analyticity. One is instantly in a quick-sand of ambiguities. Cross-talk develops between partial waves, making it difficult to separate related partial waves, for example, $J^{P C}=0^{++}, 1^{--}, 2^{++}$, and so forth, and likewise $0^{-+}, 1^{++}, 2^{-+}$, and so forth. Results which deviate significantly from analyticity cannot be believed. A further difficulty is that energy independent analysis requires literally thousands of parameters: magnitudes and phases of all amplitudes in every bin.

The converse situation in energy dependent analysis is that one must be careful to explore the maximum possible variation of $s$-dependence. For resonances, this is little problem in principle. A Breit-Wigner amplitude of constant width is appropriate unless one encounters thresholds. If these thresholds are sharp, they are easily accomodated by the Flatte form

$$
f=B(s) /\left[M^{2}-s-i\left(g_{1}^{2} \rho_{1}(s)+g_{2}^{2} \rho_{2}(s)\right)\right],
$$

for the two-channel case. Here $g$ are coupling constants and $\rho$ are phase-space factors $2 k / \sqrt{s}$, where $k$ is momentum in the decay channel. Below threshold, $\rho$ needs to be continued analytically or by means of a dispersion relation. In the numerator, $B(s)$ is a centrifugal barrier factor needed in most partial waves; form factors usually have negligible effect over the widths of known resonances.

Complications arise however where the threshold opens for production of a final state with significant width, for example, $f_{0}(1370) \rightarrow 4 \pi$. There is still a well defined prescription: $\rho(s)$ needs to be integrated over the phase space of the exit channel. In addition, $M^{2}-s$ needs modification to $M^{2}-s-m(s)$, where

$$
m(s)=\frac{s-M^{2}}{\pi} \int \frac{d s^{\prime} M \Gamma_{4 \pi}\left(s^{\prime}\right)}{\left(s^{\prime}-s\right)\left(s^{\prime}-M^{2}\right)} .
$$


Evaluation of the dispersion integral needs to be done only once if the form of $\Gamma_{4 \pi}$ is known, but the program then needs to interpolate in a table of values; in practice this is trivial.

It is sometimes argued that "backgrounds" may be needed in every partial wave. This point needs clarification. Left-hand cuts for each partial wave generate slowly varying driving forces. However, in the $N / D$ approach, these are isolated in the $N$ function. The denominator $D$ contains the phase information concerning resonances. It is not necessary to add separately left-hand cuts as "backgrounds": these are already built into resonances. The classic example is Chew-Low theory [34], where nucleon exchange drives the $\Delta(1232)$ and alters its line-shape from a simple Breit-Wigner by an amount which can in practice be used to make an accurate determination of the $\pi N N$ coupling constant.

There may of course be broad components, for example, hybrids or molecular states, in addition to the regular $3 q$ states, and one needs to keep a watchful eye open for such broad components.

The virtue of energy dependent analysis is that the number of fitted parameters is reduced to a few per partial wave. This has the advantage that programmes run quickly. The downside is that one must be careful not to miss something, requiring time to explore the options. This procedure must be viewed as a process of successive approximation.

The proof of the pudding is in the results. Energy dependent analysis has dug out of Crystal Barrel data a regular array of resonances. Their star-rating can be investigated by varying their parameters and dropping them completely from the analysis to see what happens. Analyses below the $\bar{p} p$ threshold have mostly been done with energy independent analysis. The result, however, is a number of missing states which can be confidently predicted from the quark model, notably low spin states with $J^{P C}=0^{-+}, 1^{++}$, and $2^{--}$. My opinion is that these would emerge with judicious use of analytic forms for amplitudes. That needs to be done with existing data.

\subsection{Possible $I=2$ contributions}

There is one remaining issue which goes beyond current partial wave analyses. The $\pi \pi$ isospin 2 amplitude may contribute, though there is presently no evidence for this. If it does, its Clebsch-Gordan coefficients differ between $\pi^{+} p \rightarrow \pi^{+} \pi^{+} n$ and $\pi^{+} p \rightarrow \pi^{+} \pi^{0} p$ final states, for example. To investigate this possibility, it is desirable to take some data for the reactions in the bottom part of Table 1 . This can be done from a liquid hydrogen target using $1 \mathrm{C}$ kinematic fits for these reactions. The alternative is to use a time-of-flight spectrometer, giving a $2 \mathrm{C}$ fit.

\section{Conclusions}

It is technically straightforward to use a frozen-spin target in a $4 \pi$ detector to collect data on baryon and light-meson spectroscopy. Such a program could be completed in a few years and would expand enormously the reliability and extent of available data. Data on $\pi N$ would strengthen the results which can be deduced from the existing photoproduction data (plus the measurements forseen at ELSA with polarised target and polarised photons).

From such a program, it is predictable that the spectroscopy of the regular baryons and mesons could be determined completely up to $2400-2500 \mathrm{MeV}$, that is, over two radial excitations, which is surely sufficient to see the picture. Once this spectrum is established, the door is open to uncovering glueballs with confidence in BES III data on decays of $J / \Psi, \Psi^{\prime}$ and $\Psi^{\prime \prime}$. 
It is very likely that the outlines of the hybrid spectrum would also materialise. In meson spectroscopy, there are presently three good candidates: the well known $\pi_{1}(1600)$ with exotic quantum numbers $J^{P C}=1^{-+}$, as well as two $2^{-+}$states $\pi_{2}(1880)$ and $\eta_{2}(1870)$, with masses (and decay modes) which do not fit regular $q \bar{q}$ states. In addition the $\pi(1800)$ has decay modes characteristic of those to be associated with a hybrid; the problem here is that the $q \bar{q}$ state expected at $\sim 1650 \mathrm{MeV}$ from the Regge trajectories of Figure 2(c) is as yet unknown and could be the $\pi(1800)$.

The cost of such a program in terms of new equipment is small, and it would indeed extend the lives of existing high-quality detectors. A significant effort of man-power is, however, required in partial wave analysis.

\section{Acknowledgment}

The author wishes to thank Leonid Glozman, Sergey Afonin, and Andrei Sarantsev for extensive discussions of both theoretical and experimental topics.

\section{References}

[1] W.-M. Yao, et al., "Particle data group," Journal of Physics , vol. 33, no. 1, 2006.

[2] A. Starostin, et al., "Crystal ball collaboration," Physical Review C , vol. 67, no. 6, 2003.

[3] G. Penner and U. Mosel, "Vector meson production and nucleon resonance analysis in a coupledchannel approach for energies $m_{N}<\sqrt[s]{<2} \mathrm{GeV}$. I. Pion-induced results and hadronic parameters," Physical Review C , vol. 66, no. 5, p. 43 pages, 2002, Article ID 055211.

[4] S. Prakhov, B. M. K. Nefkens, C. E. Allgower, et al., "Measurement of $\pi^{-} p \rightarrow \pi^{0} \pi^{0} n$ from threshold to $p_{\pi^{-}}=750 \mathrm{MeV} / \mathrm{c}$," Physical Review C , vol. 69, p. 15 pages, 2004, Article ID 045202.

[5] S. Prakhov, B. M. K. Nefkens, C. E. Allgower, et al., "Reaction $K^{-} p \rightarrow \pi^{0} \pi^{0} \Lambda$ from $p K^{-}=514$ to 750 $\mathrm{MeV} / c^{\prime}$, Physical Review C , vol. 69, p. 4 pages, 2004, Article ID 042202.

[6] D. M. Manley and E. M. Saleski, "Multichannel resonance parametrization of $\pi N$ scattering amplitudes," Physical Review D , vol. 45, no. 11, pp. 4002-4033, 1992.

[7] D. H. Saxon, R. D. Baker, K. W. Bell, et al., "The reaction $\pi^{-} p \rightarrow K^{0} \Lambda^{0}$ up to $2375 \mathrm{MeV} / c$ new results and analysis," Nuclear Physics B , vol. 162, no. 3, pp. 522-546, 1980.

[8] A. V. Sarantsev, et al., "New results on the Roper resonance and the $P_{11}$ partial wave," http://arxiv.org/abs/0707.3591.

[9] A. V. Anisovich, V. Kleber, E. Klempt, V. A. Nikonov, A. V. Sarantsev, and U. Thoma, "Baryon resonances and polarization transfer in hyperon photoproduction," http://arxiv.org/abs/0707.3596.

[10] V. A. Nikonov, A. V. Anisovich, E. Klempt, A. V. Sarantsev, and U. Thoma, "Further evidence for $\mathrm{N}(1900) P_{13}$ from photoproduction of hyperons," http://arxiv.org/abs/0707.3600.

[11] U. Thoma, M. Fuchs, A. V. Anisovich, et al., " $N^{*}$ and Delta* decays into N pi0 pi0," http://arxiv.org/abs/0707.3592.

[12] R. Bradford, R. A. Schumacher, G. Adams, et al., "First measurement of beam-recoil observables $C_{x}$ and $C_{z}$ in hyperon photoproduction," Physical Review C, vol. 75, p. 25 pages, 2007, Article ID 035205.

[13] A. V. Anisovich, C. A. Baker, C. J. Batty, et al., "I = 0, C = +1 mesons from 1920 to $2410 \mathrm{MeV}$," Physics Letters B , vol. 491, no. 1-2, pp. 47-58, 2000.

[14] D. V. Bugg, "Four sorts of meson," Physics Reports , vol. 397, pp. 257-358, 2004.

[15] E. Klempt, "Mass formula for baryon resonances," Physical Review C , vol. 66, no. 5, p. 4 pages, 2002, Article ID 058201.

[16] A. V. Anisovich, C. A. Baker, C. J. Batty, et al., "Partial wave analysis of $\bar{p} p$ annihilation channels in flight with $I=1, C=+1$," Physics Letters B , vol. 517, no. 3-4, pp. 261-272, 2001.

[17] A. V. Anisovich, C. A. Baker, C. J. Batty, et al., "Combined analysis of meson channels with $I=1$, $C=-1$ from 1940 to $2410 \mathrm{MeV}$," Physics Letters B , vol. 542, no. 1-2, pp. 8-18, 2002.

[18] A. V. Anisovich, C. A. Baker, C. J. Batty, et al., " $I=0, C=-1$ mesons from 1940 to $2410 \mathrm{MeV}$," Physics Letters B , vol. 542, no. 1, pp. 19-28, 2002. 
[19] L. Ya. Glozman, "Restoration of chiral and $U(1)_{A}$ symmetries in excited hadrons," Physics Reports , vol. 444, no. 1, pp. 1-49, 2007.

[20] S. S. Afonin, "Properties of possible new unflavored mesons below $2.4 \mathrm{GeV}$," Physical Review C , vol. 76, p. 5 pages, 2007, Article ID 015202.

[21] R. L. Jaffe, D. Pirjol, and A. Scardicchio, "Parity doubling and $S U(2)_{L} \times S U(2)_{R}$ restoration in the hadron spectrum," Physical Review Letters , vol. 96, 2006, Article ID 121601.

[22] R. L. Jaffe, D. Pirjol, and A. Scardicchio, "Pion decoupling and $S U(2)_{L} \times S U(2)_{R}$ restoration in the hadron spectrum," Physical Review D , vol. 74, p. 1 page, 2006, Article ID 057901.

[23] M. Shifman and A. Vainstein, "Highly excited mesons, linear Regge trajectories and the pattern of the chiral symmetry realization," http://arxiv.org/abs/0710.0863.

[24] L. Ya. Glozman and A. V. Nefediev, "Chiral symmetry and the string description of excited hadrons," http://arxiv.org/abs/0704.2673.

[25] S. S. Afonin, "Parity doubling in particle physics," to appear in International Journal of Modern Physics A.

[26] S. S. Afonin, "Evidence for new baryon like symmetries in meson spectrum," http://arxiv.org/ abs/0707.1291.

[27] D. S. Carman, "GlueX: the search for gluonic excitations at Jefferson laboratory," in AIP Conference Proceedings, vol. vol. 814, pp. 173-182, Rio de Janeiro, Brazil, 2005.

[28] D. V. Bugg and D. Axen, "Y* resonances in the mass range $1520-2430 \mathrm{MeV} / \mathrm{c}^{2}$," Z. Phys. C - Particles and Fields, vol. 46, pp. S31-35, 1990.

[29] C. E. Waltham, R. Shypit, D. A. Axen, et al., "Spin-spin correlations and spin asymmetries for the reaction $\vec{p} \vec{p} \rightarrow p n \pi^{+}$at intermediate energies," Nuclear Physics A, vol. 433, no. 4, pp. 649-670, 1985.

[30] R. L. Shypit, D. V. Bugg, A. H. Sanjari, et al., "Spin dependence of $p p \rightarrow n p \pi^{+}$from 492 to $796 \mathrm{MeV}$," Physical Review C , vol. 40, no. 5, pp. 2203-2217, 1989.

[31] E. Eisenhandler, W. R. Gibson, C. Hojvat, et al., "Measurement of differential cross sections for antiproton-proton annihilation into charged pion and kaon pairs between 0.79 and $243 \mathrm{GeV} / \mathrm{c}$," $\mathrm{Nu}$ clear Physics B , vol. 98, no. 1, pp. 109-154, 1975.

[32] A. Hasan, D. V. Bugg, J. R. Hall, R. L. Shypit, et al., "Differential cross sections and analysing powers for $\bar{p} P \rightarrow \pi^{-} \pi^{+}$and $K^{-} K^{+}$from 360 to $1550 \mathrm{MeV} / \mathrm{c}^{\prime \prime}$ Nuclear Physics B , vol. 378, no. 1-2, pp. 3-51, 1992.

[33] K. Peters, "Hadron physics at FAIR," in AIP Conference Proceedings, vol. vol. 814, pp. 33-41, Darmstadt, Germany, February 2006.

[34] G. F. Chew and F. E. Low, "Theory of photomeson production at low energies," Physical Review , vol. 101, no. 5, pp. 1579-1587, 1956. 

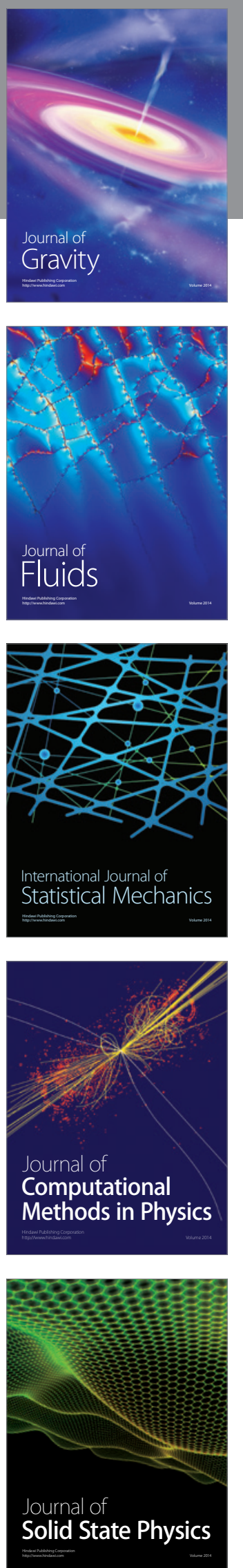

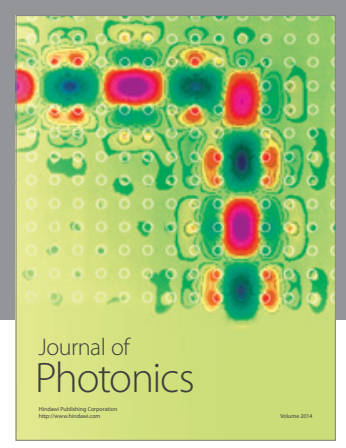

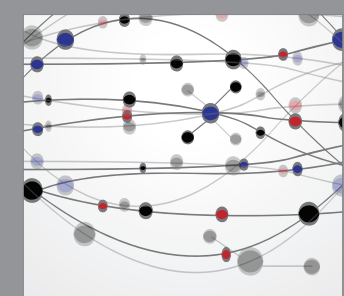

The Scientific World Journal
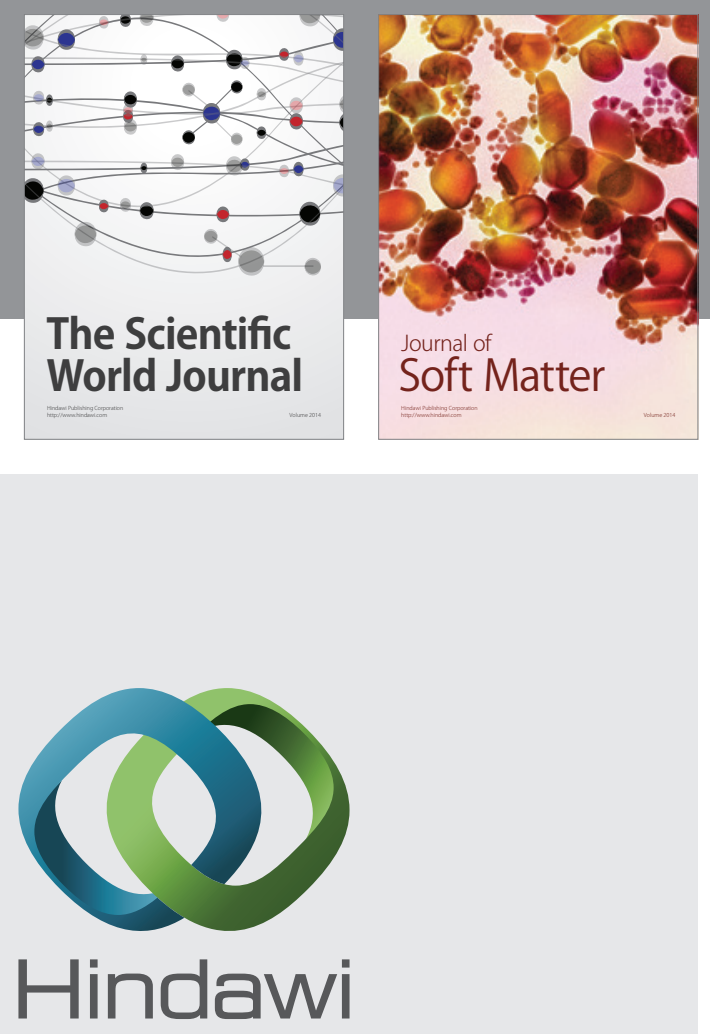

Submit your manuscripts at

http://www.hindawi.com
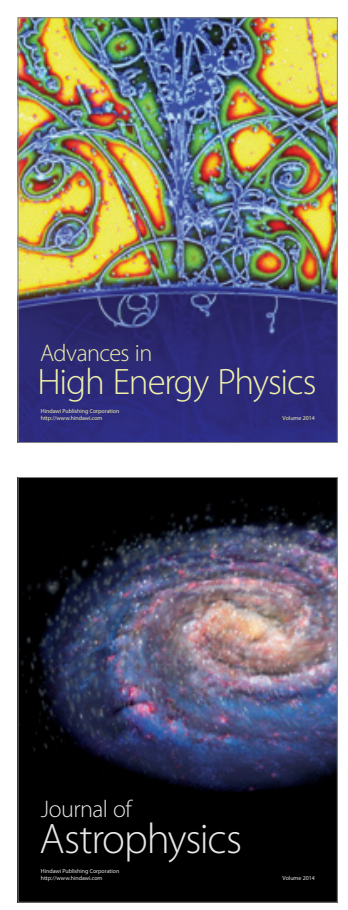
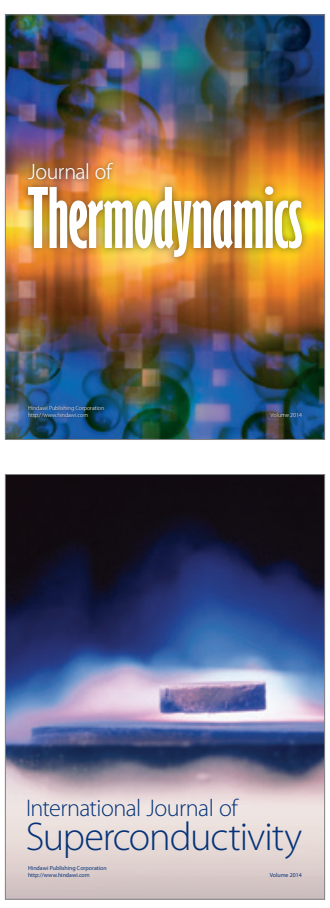
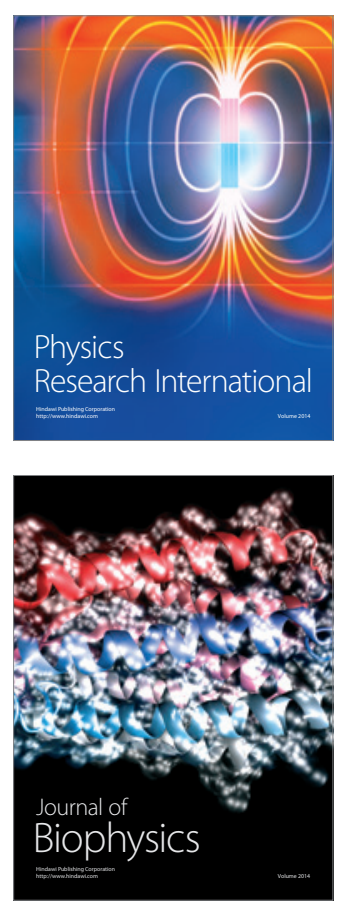
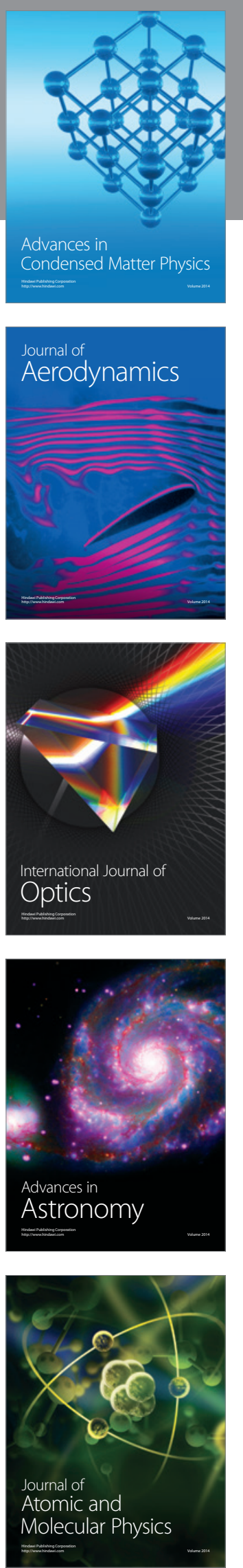